\title{
Textos fonéticos de El Salvador, Nicaragua, Costa Rica y Panamá (1960-2006)
}

\author{
Phonetic Texts from El Salvador, Nicaragua, \\ Costa Rica and Panamá (1960-2006)
}

\author{
FÉLIX FERNÁNDEZ DE CASTROa \\ ${ }^{a}$ Universidad de Oviedo, Departamento de Filología Española, España. \\ Correo electrónico: felixfer@uniovi.es
}

La dialectología, la geografía lingüística y la sociolingüística han recurrido con frecuencia a la transcripción fonética de textos para ilustrar los modos de hablar analizados en las monografías dialectales, los atlas lingüísticos y los estudios variacionistas. Este artículo estudia seis de estos trabajos sobre el espańol de El Salvador, Nicaragua, Costa Rica y Panamá que se publicaron entre 1960 y 2006. Se describe la obra en que aparecen los textos fonéticos (con una muestra de cada uno), su marco metodológico y el sistema de transcripción empleado.

Palabras clave: español en América, fonética, dialectología, historia de la lingüística.

In Dialectology, Geographical Linguistics and Sociolinguistics, phonetic transcription is often used to illustrate the speech analyzed in monographs, linguistic atlases and variation studies. This paper studies six such works dealing with El Salvador, Nicaragua, Costa Rica and Panamá, published between 1960 and 2006. It describes the work in which each phonetic text is included (with a sample of the transcription), its theoretical framework and the system of phonetic notation employed by each author.

Key words: American Spanish, Phonetics, Dialectology, Historiography of Linguistics. 


\section{INTRODUCCIÓN}

Este artículo es una entrega más de la labor de recuperación y edición llevada a cabo en el proyecto "Antología de Textos Fonéticos Hispano-Americanos" (en adelante, ATeFonHA $)^{1}$. En publicaciones anteriores se han presentado los objetivos y metodología generales del trabajo, una selección de materiales de una decena de países, y estudios particulares de los textos de Puerto Rico, República Dominicana y $\mathrm{Cuba}^{2}$.

\subsection{El español centroamericano: algunos logros recientes}

En 2008 el profesor costarricense Miguel Ángel Quesada Pacheco publicó un detallado status quaestionis sobre el estudio del español hablado en América Central ${ }^{3}$. Su informe recopila lo conocido —escaso, y más normativista que descriptivo-, lo sitúa en su marco de procedencia, y determina qué lagunas debían cubrirse. Para el autor, el mayor problema había sido y seguía siendo la descoordinación de los esfuerzos particulares aislados, y de ahí su llamamiento a trabajar de forma colegiada, con publicaciones, congresos y proyectos que integraran la labor de todos los estudiosos.

Pues bien, a día de hoy, y en gran medida por el buen hacer académico y científico de este investigador, nuestro conocimiento del español centroamericano tiene fundamentos bien firmes, y con un nivel de coordinación y detalle superior al de otras regiones hispanohablantes. Bajo la cobertura de su proyecto Atlas Lingüistico de América Central $(A L A C)$, y en los tres lustros apenas transcurridos desde su llamado en Managua, él y sus equipos de investigadores han realizado ya al menos dos recopilaciones de estudios sobre el nivel fonético y el morfosintáctico (Quesada 2010a y 2013), y una docena de piezas que se reparten los diferentes países y niveles estudiados hasta el momento: Costa Rica, Nicaragua, Panamá, El Salvador, Belice y Honduras en los planos fonético, gramatical, léxico y etnográfico, y Guatemala al menos en el primero de ellos ${ }^{4}$. Además, de acuerdo con la aspiración original de quien las ha iniciado y coordinado, todas estas aportaciones

\footnotetext{
${ }^{1}$ En esta fase de la investigación fue decisiva la ayuda concedida por la Universidad de Oviedo al proyecto UNOV-06-MA-501-1. También quiero dejar constancia de mi agradecimiento al investigador principal de este equipo, el profesor Álvaro Arias Cabal, quien, además de integrarme en él, me consiguió, durante una estancia de investigación en Costa Rica, una reproducción de Rodríguez Rojas (1992), trabajo que, por su condición de inédito, difícilmente hubiera podido obtener yo por mis medios. Esta gratitud se extiende al profesor Víctor M. Sánchez Corrales, director de la tesis, quien se la proporcionó. Por último, deseo añadir mi reconocimiento a los revisores de $E F$, por una corrección tan minuciosa en los detalles como generosa en la comprensión y valoración de este proyecto en su conjunto.

${ }^{2}$ Fernández de Castro 2007, 2010, 2011 y 2022, respectivamente.

${ }^{3}$ Quesada 2008. El artículo recoge su ponencia en un simposio celebrado en Managua en 2006.

${ }^{4}$ Belice: Cardona (2012) y Rivera (2013); Costa Rica: Quesada (2010a); El Salvador: Rivera (2011) y Azcúnaga (2012); Guatemala: Utgård (2006) y Chavarría \& Quesada (in fieri); Honduras: Hernández (2013a, 2013b) y Ventura (2013); Nicaragua: Chavarría \& Rosales (2010); Panamá: Tinoco (2010) y Cardona (2012).
} 
de geografía lingüística presentan la misma estructura y se han obtenido con la misma metodología, para permitir así la comparación y el procesamiento ordenados de los datos particulares.

El presente artículo aspira a ser un muy pequeño complemento a tales logros, con la recuperación de materiales descriptivos que se hallan a veces perdidos o semiolvidados, pero que pueden quizá servir de ilustración textual al moderno conocimiento de las hablas de la región.

\subsection{Mapa y tabla resumen}

Presentaré en estas páginas seis trabajos de siete autores que contienen un total de 34 textos fonéticos, transcritos a 31 informantes de 10 lugares distintos, y que suman unas 28.000 palabras. El mapa y la tabla siguientes los muestran de forma sinóptica. En el primero figuran solo referencias numéricas, a lugares o áreas, especificadas después en la tabla. Si un autor ha compilado muestras de varias procedencias (caso de Canfield o Robe, por ejemplo), aquí se separan y se vinculan a sus respectivos emplazamientos. Cada fila muestra la clave y el lugar, la referencia del trabajo y el bloque particular de textos (autor, año y páginas), el número de informantes, el de textos, el cómputo de palabras (exacto en textos pequeños, estimado las más veces por extrapolación), y el tipo y nivel de detalle de cada transcripción.

Respecto a las modalidades de representación fonética, en las introducciones de cada estudio se hablará más de ellas. Baste anticipar ahora que las propiamente fonéticas se distinguen por emplear, bien el Alfabeto de la Revista de Filología Espańola (ARFE) o el Alfabeto Fonético Internacional (AFI), con diferentes niveles de detalle (ancho, semiestrecho, estrecho). Pero hay más. Como ya se ha explicado en anteriores entregas de este proyecto, muchos lingüistas han aportado textos que, pese a no ser transcripciones sensu stricto, vale la pena incluir en esta antología 5 . En el caso de Costa Rica, Solano (1986), por ejemplo, no usa un alfabeto fonético, pero consigue con su transcripción ortográfica reflejar abundantes fenómenos fonéticos y morfológicos de interés, por lo que sus textos se presentarán aquí con la etiqueta "Ortografía Fonetizada" (OF).

\footnotetext{
${ }^{5}$ Estos criterios se explican con detalle en Fernández de Castro (2007: 292-293).
} 
Figura 1. Mapa con la distribución geográfica de los textos.

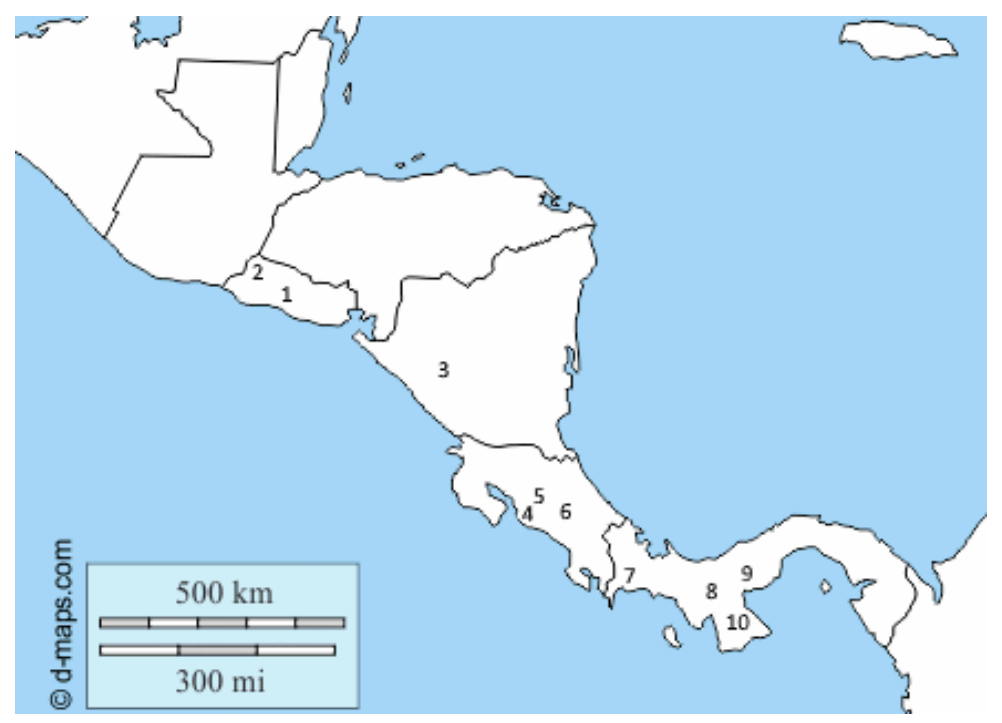

Tabla 1. Descripción de los materiales presentados en el mapa

\begin{tabular}{|c|c|c|c|c|c|c|c|}
\hline N.o & Lugar & Referencia & Sujetos & Textos & \multicolumn{2}{|c|}{ Palabras } & Formato \\
\hline \multicolumn{8}{|c|}{ El Salvador } \\
\hline- & No especificado & Canfield 1960: 58-64 & 4 & 2 & 650 & * & ARFE ancho manuscrito \\
\hline 1 & Santa Ana & Canfield 1960: 54-56 & 1 & 2 & 370 & * & ARFE ancho manuscrito \\
\hline 2 & Candelaria & Canfield 1960: 56-58 & 2 & 1 & 270 & * & ARFE ancho manuscrito \\
\hline \multicolumn{8}{|c|}{ Nicaragua } \\
\hline 3 & Granada & Quilis 2002: 19-27 & 1 & 1 & 291 & & AFI estrecho \\
\hline \multicolumn{8}{|c|}{ Costa Rica } \\
\hline 4 & Puntarenas & Rodríguez 1992: 77-152 & 9 & 9 & 24.000 & * & AFI semiestrecho \\
\hline 5 & Palmares & Solano 1986: 140-142 & 1 & 1 & 2.200 & * & OF \\
\hline 6 & San José & Pińeros 2006 & 1 & 1 & 119 & & AFI estrecho \\
\hline \multicolumn{8}{|c|}{ Panamá } \\
\hline 7 & David & Piñeros 2006 & 1 & 1 & 119 & & AFI estrecho \\
\hline 8 & Varaguas & Robe 1960: 158 & 6 & 6 & 90 & & Mixto ARFE-AFI \\
\hline 9 & Coclé & Robe 1960: 157 & 3 & 4 & 105 & & Mixto ARFE-AFI \\
\hline 10 & Los Santos & Robe 1960: 157-158 & 2 & 6 & 95 & & Mixto ARFE-AFI \\
\hline \multicolumn{3}{|c|}{ Totales: 10 lugares especificados y 6 autores } & 31 & 34 & 28.300 & ${ }^{\top}$ & \\
\hline
\end{tabular}

* Cantidad estimada. 


\subsection{Estructura de este trabajo}

Los bloques dedicados a cada estudio tendrán una estructura repetida, formada por cuatro elementos: presentación, facsimil, transcripción ortográfica y transcripción fonética adaptada. Cada una de ellas juega un papel específico. La extensión media de los textos escogidos como muestra es de unas 150 palabras.

La presentación sitúa las coordenadas metodológicas de las que surgió cada trabajo y la escuela o influencias teóricas perceptibles en la obra de su autor. Se describirá su sistema de transcripción, y se dará el perfil del informante de la muestra escogida, cuando esté disponible.

El facsimilse ha obtenido, cuando ha sido posible, de ediciones originales, procurando buscar siempre la más antigua y mejor conservada, y realizando una digitalización en calidad profesional. Algún caso procede aún de una copia, pero la tendencia es conseguir el mayor grado posible de fidelidad al origen.

La transcripción ortográfica presenta el texto en ortografía normativa del español. Si bien es frecuente que los propios autores ofrezcan esta versión de los textos que transcriben, hay excepciones. Cuando no lo hacen, se ha realizado aquí una reconstrucción basada en la versión fonética. Si hay discordancias entre lo transcrito fonéticamente y su equivalente ortográfico, se recompone la transcripción ortográfica para hacerla coherente y evitar desajustes de interpretación, dejando constancia de tal remodelación.

El apartado final, el titulado transcripción fonética adaptada, es el más comprometido de esta antología. Hacer una versión modificada de una transcripción fonética es tarea difícil de afrontar. Quien la adapta no ha escuchado la emisión originaria (aunque en algún caso moderno empieza a ser posible, gracias al empleo de tecnología multimedia en la presentación de los materiales). ¿Qué puede legitimar, pues, que se atreva a retocarla? Únicamente la certeza de que las ventajas superen a los inconvenientes. En estas versiones se ha buscado presentar una versión formalmente homogeneizada de todos los originales. Quien recorra los facsímiles encontrará notaciones en AFI y en ARFE más o menos ortodoxas, tipográficas o manuscritas, y las hallará en nivel ancho, semiestrecho o estrecho.

Para poder aprovechar aportaciones tan dispares como un corpus unificado, se han convertido todas (salvo la ortográfico-fonética de Solano 1986), en el último apartado de cada sección, en transcripciones semiestrechas en $\mathrm{AFI}^{6}$. Esto quiere decir que se renuncia a detallar las variantes tímbricas de las vocales, salvo las que entran en diptongo; y que, de las variaciones consonánticas inducidas por el contexto fónico, se han reflejado solo las más generales, más los rasgos privativos y característicos de las hablas centroamericanas. El resultado es un formato homogéneo, apto para un análisis que abarque el nivel fonético. En general, los textos resultan de simplificar transcripciones inicialmente más complejas. Todos presentan una disposición parecida, independiente de la original, y se han vertido

\footnotetext{
${ }^{6}$ Para los criterios de aplicación se han seguido las pautas emanadas recientemente del C.S.I.C., presentes por ejemplo en García Mouton (2015) y García Mouton \& Molina Martos (2017).
} 
en una misma tipografía, la fuente de libre acceso Doulos SIL IPA, versión 4.106, de SIL International.

Por estas razones, y con plena conciencia del coeficiente de distorsión que implican, se han introducido aquí estas versiones adaptadas, siempre junto al facsímil como referente de verificación.

Se trataría pues de articular el acceso al texto en varios niveles: el mapa y la tabla preliminares son una guía para llegar a todos los materiales disponibles, desglosados por localizaciones; con el texto ortográfico entramos directamente al léxico y la gramática; con el fonético adaptado accedemos al plano de la expresión de una forma normalizada; finalmente, el investigador podría construir, con los facsímiles y los datos de las presentaciones, su propio corpus textual del español centroamericano.

\section{CANField 1960}

En el legado del hispanista norteamericano Delos Lincoln Canfield destaca especialmente su monografía sobre la fonética del español en América, obra cuyo desarrollo se prolongó durante décadas (Canfield 1962, 1981 y 1988). Este trabajo, que presenta las variantes de pronunciación país a país, no se entiende sin la previa acumulación de estudios parciales ceñidos a diversas regiones de la América hispanohablante, apoyados en las observaciones recogidas durante sus expediciones dialectológicas por todo el continente (Beardsley 1993). Y de entre estas investigaciones particulares sobresale, por su extensión y por lo ambicioso de sus planteamientos, la que aquí se presenta en extracto, el artículo que Canfield dedicó al español de El Salvador, publicado en 1960. Un estudio que, pese a su formato, es casi una monografía en sí mismo.

Aunque la trayectoria formativa y profesional de Canfield se desarrolló por completo en los Estados Unidos (más un amplio recorrido como profesor invitado por Espańa y la América hispana), el autor mostró durante toda su vida científica un marcado apego a principios y métodos directamente trasplantados de la Escuela de Madrid, en especial a través del magisterio norteamericano de Tomás Navarro Tomás. Las primeras líneas del trabajo aquí recordado, por ejemplo, se dedican a colocarlo en la estela de las investigaciones surgidas del modelo del Cuestionario lingüistico hispanoamericano (Navarro 1945). Canfield usó siempre para sus transcripciones el alfabeto fonético de la Revista de Filología Española, incluida la semiestrecha mostrada aquí, que se añade, manuscrita, como complemento de la descripción hecha en el estudio, también al modo de Navarro. Y, sobre todo, la concepción de fondo de la filología y de la investigación lingüística de Canfield fue siempre profundamente historicista. En fecha tan adelantada como 1988, cuando el estructuralismo y la sociolingüística habían pasado sucesivamente sobre la antigua dialectología, aún sostiene con firmeza Canfield que "...casi la totalidad de la fonología del español de América se remonta a etapas en la evolución del castellano andaluz de la época colonial..." (Canfield 1988: 7). 
Su estudio sobre el español salvadoreño se basa en observaciones recogidas en los veranos de 1951 y 1952. Utilizó como base de trabajo la Universidad de El Salvador, y viajó desde ella a distintas regiones del país. Canfield realizó un centenar de grabaciones, en las cuales se propuso además recoger muestra de distintos sectores de la sociedad y niveles de edad. Solo de catorce sujetos da una filiación completa, y ninguno de ellos parece ser el informante origen del texto aquí escogido, de quien solo se nos dice que “...es la relación de un episodio por otro chófer" (Canfield 1960: 54).

\subsection{Facsimil (Canfield 1960: 58-59)}

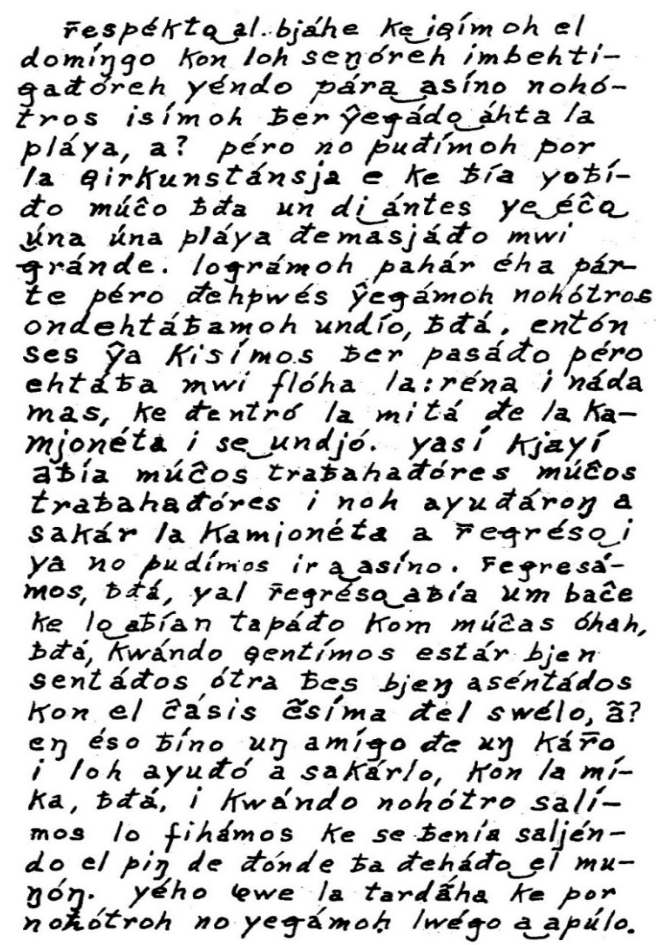

\subsection{Transcripción ortográfica}

El viaje del domingo

1 Respecto al viaje que hicimos el domingo con los señores investigadores, 2 yendo para Asino, nosotros quisimos haber llegado hasta la playa, ¿ah? Pero 3 nopudimosporlacircunstanciadequehabíallovidomucho, ¿verdad? undíaantes, 4 y es hecho una una playa demasiado muy grande. Logramos pasar esa parte 5 perodespués llegamosnosotros donde estábamoshundidos, ¿verdad? Entonces 
6 ya quisimos haber pasado pero estaba muy floja la arena y nada más-que dentró

7 la mitad de la camioneta y se hundió.-Y así que allí había muchos trabajadores,

8 muchos trabajadores, y nos ayudaron a sacar la camioneta a regreso, y ya no

9 pudimos ir a Asino. Regresamos, ¿verdad? y al regreso había un bache que lo

10 habían tapado con muchas hojas, ¿verdad? cuando sentimos estar bien sentados

11 otra vez - bien asentados con el chasis encima del suelo, $¿$ ah? ${ }^{7}$

\subsection{Transcripción fonética adaptada}

1 [ res'pekto al 'bjahe ke i' $\theta$ imoh el do'mingo kon loh se'noreh imbehtiya'ðoreh |

2 'jendo 'para a'sino | no'hotros i'simoh $\beta$ er Fje' yado 'ahta la 'plaja $\mid$ a $\uparrow||$ 'pero

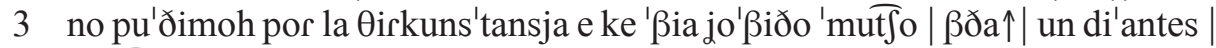

4 je 'et 50 'una 'una 'plaja ðema'sjaðo mwi 'yrande $\|$ lo'yramoh pa'har 'eha 'parte

5 'pero ðeh'pwes fje' yamoh no'hotros ondeh'taßamoh un'dio $\left|\beta^{\prime} ð a \uparrow\right| \mid$ en'tonses

6 jjaki'simosßer pa'saðo 'pero eh'taßa mwi'floha la:'renai'nada mas|keðen'tro

7 la mi'ta ðe la kamjo'neta i se un'djo || ja'si kja'ji a'ßia 'mutfos traßaha'ðores |

8 'mutyos traßaha'ðores | i noh aju'ðaron a sa'kar la kamjo'neta a re'yreso i ja no

9 pu'dimos ir a a'sino || reyre'samos $\left|\beta^{\prime} ð a \uparrow\right|$ jal re'yreso a'ßia um batfe ke lo

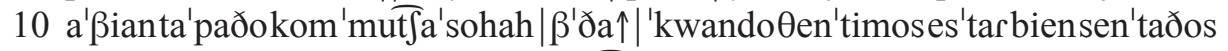

11 'otra ßes | bjey a'sen'tados kon el 'T $\mathrm{t}$ asis ẽ'sima ðel 'swelo $\mid \tilde{a} \uparrow]^{8}$

\section{Quilis y Quilis SANZ 2002}

La bibliografía americana de Antonio Quilis, a partir del momento en que se inician los trabajos del Atlas Lingüistico de Hispanoamérica, se va llenando de títulos referidos a lugares como Nicaragua, Panamá o Belice, por un lado, y Ecuador y Bolivia, por otro. Centroamérica y la zona andina fueron, en efecto, los dos ámbitos a los que volvió ya constantemente, para investigar y recoger datos, y ojalá algún día podamos conocer el resultado de estos trabajos, que su prematuro e inesperado fallecimiento dejó inéditos.

Quilis, muy ligado al Consejo Superior de Investigaciones Científicas, y por tanto continuador del espíritu de la Escuela de Madrid, fue un gran cultivador del ejercicio de la transcripción fonética. Pero además fue uno de los más importantes fonetistas de nuestra época, y eso hace aún más valioso cada texto conservado en alguna de sus páginas.

Aquí se muestra uno de ellos, accesible gracias a que apareció en un pequeño Cuaderno de Prácticas editado por la U.N.E.D. para complementar el programa de su asignatura de Español de América. En él presenta Quilis, muy sucintamente, dos comentarios

\footnotetext{
${ }^{7}$ Transcripción original del autor, completada con algunos segmentos para restablecer la plena correspondencia con la versión fonética.

${ }^{8}$ En esta adaptación se han añadido las pausas que indica el autor en su versión ortográfica.
} 
de textos orales (es decir, de dos transcripciones fonéticas hechas por él, una de Nicaragua y otra de Bolivia) y otro de un fragmento literario. Se trata de dos de las transcripciones más detalladas y cuidadas que se han hecho nunca del español americano, plasmadas con AFI estrecho e indicaciones prosódicas añadidas. De la primera de ambas, recogida en este apartado, hace el autor la siguiente presentación:

El siguiente texto, que fue grabado en cinta magnetofónica, es de un médico puericultor, de 58 ańos, natural de Granada, Nicaragua. El diálogo se inicia con la pregunta que le formula el encuestador sobre si en Nicaragua al murciélago le llaman también "vampiro"; el informante explica que el vampiro es diferente, es el hematófago. (Quilis \& Quilis Sanz 2002: 19)

\subsection{Facsimil (Quilis \& Quilis Sanz 2002: 20-21)}

- ¿Y éste muerde o cómo hace?

dzó e: $\uparrow$ la la e enperjénsja $\uparrow$ jó la túße $\downarrow$ nó $\uparrow$ pwehé é đesír $\mathrm{e} \rightarrow \mathrm{I}$ púse a la pessóna i $\uparrow$

jó mehkondí $\uparrow$ para $\rightarrow \mid$ para ßé ké lo ke asía $\downarrow$ l jel anĩmál éntra $\uparrow$ I

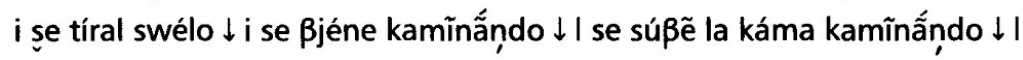

i k'on el ála $\uparrow$ leßán,ta el mohyitéro $\uparrow$ si [tjéne] mokitéro $\downarrow$ l

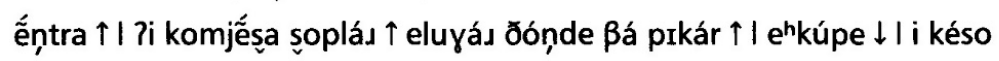

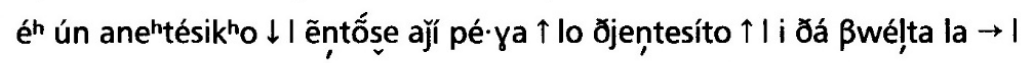

la tró:mpa $\downarrow$ l atasér $\uparrow \mid$ ?û́n sakaßokáoo $\downarrow$ l [...] se đfúpa la sấye $\uparrow$

sấye nó șe koayúla $\downarrow$ l kwan, do ehtá $\rightarrow$ múi $\uparrow$ I T̃upáo đe șấye $\uparrow$ ẽn,

se ßjéne đegahán,do é $\downarrow$ li ata ata ká $\downarrow \downarrow$ l de kómo e entá e pesáo $\downarrow$ al ráto

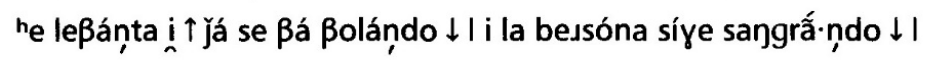

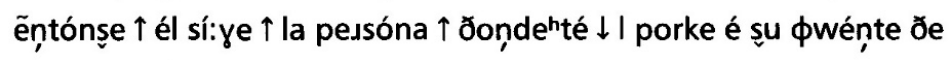

alimẽntasjốn $\downarrow$ I sja la pessóna la kítan dénte kwártọe $\uparrow$ la ßá $\rightarrow$ I

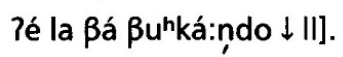

\subsection{Transcripción ortográfica}

El murciélago hematófago o 'vampiro'

— ¿Y este muerde o cómo hace?

1 Yo, e... la experiencia yo la tuve ¿no?, pues he de decir, e... puse a la

2 persona y yo me escondí para, para ver qué es lo que hacía: [y] el

3 animal entra y se tira al suelo y se viene caminando. Se sube en la cama 
4 caminando. Y con el ala, levanta el mosquitero, si [tiene] mosquitero. 5 Entra, y comienza a soplar el lugar donde va a picar. Escupe, y que eso 6 es un anestésico; entonces, allí pega los dientecitos, y da vuelta a la, 7 la trompa, hasta hacer un sacabocado; [...] se chupa la sangre, 8 sangre no se coagula; cuando está muy chupado de sangre, entonces 9 se viene desgajando é, y hasta, hasta cae de cómo está de pesado; al rato

10 se levanta y ya se va volando, y la persona sigue sangrando. 11 Entonces, él sigue la persona donde esté, porque es su fuente de 12 alimentación; si a la persona la quitan de este cuarto él la va, 13 él la va buscando.

\subsection{Transcripción fonética adaptada}

- ¿Y éste muerde o cómo hace?

1 [ Fjo e: $\uparrow$ la la e $\mathrm{e}^{\mathrm{h}} \mathrm{pe}$ 'rjensja $\uparrow$ 'jo la 'tuße $\downarrow$ 'no $\uparrow$ pwe $^{\mathrm{th}} \mathrm{e}$ 'e ðe'sir e $\rightarrow \mid$ 'puse

2 a la pe.'sona i $\uparrow$ 'jo mehkon'di $\uparrow$ para $\rightarrow \mid$ para 'Be 'ke lo ke a'sia $\downarrow \mid$ jel

3 ani'ma'lentra $\uparrow \mid$ i ze 'tiral 'swelo $\downarrow$ i se ' $\beta$ jene kami'nando $\downarrow \mid$ se 'sußẽ la 'kama

4 kami'nando $\downarrow \mid \mathrm{ik}^{\mathrm{h}}$ on e'lala $\uparrow \mid$ le'ßanta el mo ${ }^{\mathrm{h}} \mathrm{yi}^{\prime}$ tero $\uparrow$ si ['tjene] moki'tero $\downarrow \mid$

5 'entra $\uparrow \mid$ ?i ko'mjẽza zo'plaı $\uparrow$ elu'ya. 'ðonde 'Ba pi'kar $\uparrow \mid \mathrm{e}^{\mathrm{h}}$ 'kupe $\downarrow \mid \mathrm{i}$ 'keso

$6 \quad \mathrm{e}^{\mathrm{h}}$ 'un ane ${ }^{\mathrm{h}}$ tesik ${ }^{\mathrm{h}} \mathrm{o} \downarrow \mid$ en'tõze a'ji' peya $\uparrow$ lo ðjente'sito $\uparrow \mid \mathrm{i}$ 'ða ' $\beta w e l t a$ la $\rightarrow \mid$ 7 la 'tro:mpa $\downarrow \mid$ ata'ser $\uparrow \mid$ 'Tun sakaßo'kaðo $\downarrow \mid[\ldots]$ se 'Tfupa la 'sãye $\uparrow$ 8 'sãye 'no ze koa'yula $\downarrow \mid$ kwando e ${ }^{\text {h' }}$ ta $\rightarrow$ 'mui $\uparrow \mid$ t fu'pao ðe 'zãye $\uparrow$ en'tõse 9 se ' $\beta$ jene ðega'hando 'e $\downarrow \mid$ i ata ata 'ka $\downarrow \downarrow \mid$ de 'komo e ${ }^{\text {h' }}$ 'ta e pe'sao $\downarrow$ al 'rato 10 he le'ßanta i $\uparrow$ 'ja se ' $\beta$ a $\beta$ o'lando $\downarrow \mid$ i la be.ı'sona 'siye say'grando $\downarrow \mid$ 11 en'tonze $\uparrow$ 'el 'sirye $\uparrow$ la pe.ı'sona $\uparrow$ ðonde ${ }^{h}$ te $\downarrow \mid$ porke 'e zu 'фwente ðe 12 alimenta'sjon $\downarrow \mid$ sja la pe.ı'sona la 'kitan 'de ${ }^{\mathrm{h}}$ te 'kwartoe $\uparrow$ la ' $\beta \mathrm{a} \rightarrow \mid$

13 '?e la ' $\beta$ a $\beta \mathrm{u}^{\mathrm{h}}$ 'ka:ndo $\downarrow \|$ ]

\section{Solano 1986}

La dialectología tradicional del español costarricense había señalado como rasgo propio de la región central el cierre tímbrico de [ - $\mathrm{e},-\mathrm{o}]$ finales en [ $-\mathrm{i},-\mathrm{u}]$ : este > esti, claro > claru (Agüero 1962: 127-128). En 1986, la profesora Yamileth Solano Rojas publicó un análisis variacionista del fenómeno, tras estudiarlo en el cantón de Palmares, provincia de Alajuela, con el que buscaba precisar los factores lingüísticos que más lo propiciaban, y la influencia de, al menos, dos variables extralingüísticas, sexo y nivel de instrucción, dado que en su opinión éstas se habían manejado de forma estereotipada e impresionística, en el pasado, para describir tales alternancias.

La población escogida para el estudio fueron cinco mujeres y cinco hombres, todos mayores de 60 años, naturales de Palmares. El factor escolaridad variaba entre la ausencia 
total o la primaria elemental, y la autora encontró un fuerte contraste entre los primeros y los segundos. En efecto, solo quienes carecían de modelos normativos se expresaron con espontaneidad y fluidez. Por el contrario, en los sujetos con una mínima instrucción actuaba como elemento distorsionador, no solo su propia conciencia normativa, sino la circunstancia de que, al ser de clase media baja, sus hijos y nietos sí habían tenido estudios medios o superiores, e imponían su censura correctora, manifestada en una mayor frecuencia de vacilaciones de uso e incluso hipercorrecciones.

El recorrido intelectual subyacente es pues parejo al que encontramos en otros muchos estudios de la época, como los de Caravedo, Ruiz Hernández o Rodríguez Rojas: la autora arranca de la tradición estructuralista y descriptivista (cita a Alarcos, Lapesa y Agüero), pero utiliza a Labov para refinar y aquilatar las observaciones heredadas, analizando con rigor estadístico qué contextos y circunstancias hacen más o menos previsible esta variación.

Sin embargo, no se queda aquí, y al igual que les ocurría a los neogramáticos del siglo XIX, algo especial llama su atención cuando describe la espontaneidad y libertad de los hablantes más iletrados, y nos ofrece como muestra un texto, en transcripción ortográfica, pero riquísima en todo tipo de detalles fonéticos y morfológicos, lo cual la hace de interés para esta compilación. Así la presenta y justifica la autora:

Hablaron [los informantes analfabetos] con mucha confianza, con fluidez, tratándonos siempre de "vos" con la mayor naturalidad. [Sus] transcripciones presentan una gran cantidad de variaciones, todas ellas de sumo interés para el lingüista, no solo la que nos ocupa. Para que quede más claro, adjuntamos una de las transcripciones de este grupo de informantes, la única mujer que habló en forma totalmente fluida, absolutamente espontánea. (Solano 1986: 138)

\subsection{Facsimil (Solano 1986: 142)}

Las vacas, casi en toas partis tenían vacas, áhi sobraba tou, onde quiera sobraba la lechi y toa sobraaa la lechi, ni se vendía, tou el mundu tenía vacas. Ah claru que sí, torrejas que llamaban y prestiñus, tou esu lu hacían. Los picaillus, viera que picaillus hacían, de meia cajuela. Esus picaillus er'hasta que le bajaba la manteca a unu por los brazus... un día de Pilar, comu un día de esta semana verdá que celebramus, si hacía bizcochu, si hacía pan, de qué nu si hacía con tantísimu huevu qui había y bartísimu... hacían de tou, ah no, no, no la via di antis, se pasaba una vida peru sabrosa... que va ahora, nunca jamás. Un güevu ahora, cuatru y meiu... y lo compraba unu a tres por peseta. Esus, los quequis los hacían de máiz di harina que llaman, el máiz, un máiz que sali arenuu, digamus di harina, entoncis los hacían di harina. 


\subsection{Transcripción ortográfica}

\section{Recuerdos de juventud}

1 Las vacas, casi en todas partes tenían vacas, áhi sobraba todo, donde quiera

2 sobraba la leche y toda sobraba la leche, ni se vendía, todo el mundo tenía

3 vacas. Ah claro que sí, torrejas que llamaban y prestiños, todo eso lo hacían.

4 Los picadillos, viera qué picadillos hacían, de media cajuela. Esos picadillos

5 era hasta que le bajaba la manteca a uno por los brazos... un día de Pilar, como

6 un día de esta semana verdad que celebramos, se hacía bizcocho, se hacía pan,

7 dequéno se hacía contantísimo huevoque habíaybaratísimo...hacían de todo,

8 ah no, no, no la vida de antes, se pasaba una vida pero sabrosa... que va ahora,

9 nunca jamás. Un huevo ahora, cuatro y medio... y lo compraba uno a tres

10 por peseta. Esos, los queques los hacían de máiz de harina que llaman, el máiz,

11 un máiz que sale aren[oso], digamos de harina, entonces los hacían de harina. ${ }^{9}$

\subsection{Transcripción ortográfico-fonética}

1 Las vacas, casi en toas partis tenían vacas, áhi sobraba tou, onde quiera 2 sobraba la lechi y toa sobraaa la lechi, ni se vendía, tou el mundu tenía 3 vacas. Ah claru que sí, torrejas que llamaban y prestiñus, tou esu lu hacían. 4 Los picaillus, viera que picaillus hacían, de meia cajuela. Esus picaillus 5 er'hasta que le bajaba la manteca a unu por los brazus... un día de Pilar, comu 6 un día de esta semana verdá que celebramus, si hacía bizcochu, si hacía pan, 7 de qué nu si hacía con tantísimu huevu qui había y bartísimu... hacían de tou, 8 ah no, no, no la via di antis, se pasaba una vida peru sabrosa... que va ahora, 9 nunca jamás. Un güevu ahora, cuatru y meiu... y lo compraba unu a tres 10 por peseta. Esus, los quequis los hacían de máiz di harina que llaman, el máiz, 11 un máiz que sali arenuu, digamus di harina, entoncis los hacían di harina.

\section{RODRíguez RoJAS 1992}

La investigadora costarricense Alejandra Rodríguez Rojas presentó en 1992 una tesis de maestría en la Universidad de Costa Rica, dirigida por Víctor M. Sánchez Corrales, cuyo objeto era el estudio fonético y fonológico del español hablado en la ciudad de Puntarenas. Al igual que Ruiz Hernández (1977) o Caravedo (1989), la autora recupera en cierto modo las autoexigencias de los fonetistas y dialectólogos tradicionales, actualizadas por los requisitos de la sociolingüística, pero con la aspiración de integrar los datos parciales en una

${ }^{9}$ Esta transcripción no figuraba en el original, y se ha elaborado aquí a partir de la versión ortográfico-fonética. 
descripción de conjunto. De hecho, en su capítulo preliminar sobre el estado de la cuestión, Rodríguez Rojas repasa los análisis variacionistas particulares que han predominado hasta entonces en la investigación de la región, y considera necesarios estudios sobre el sistema fonológico en su totalidad (1992: 11-12).

Para compensar esta carencia, la investigadora apoya su análisis en un material obtenido según las pautas de la investigación sociolingüística: nueve entrevistas libres dirigidas, grabadas a hombres y mujeres de familia oriunda de Puntarenas, con un mismo rango de edad (60 años) y nivel cultural (enseñanza primaria). Pero este corpus, cuya extensión puede estimarse en unas 24.000 palabras, es presentado íntegramente en doble transcripción, tanto ortográfica como fonética, y sobre él se construye una descripción general y muy detallada, en la que se da cuenta por ejemplo del porcentaje de aparición correspondiente a los diversos alófonos de cada entidad fonológica. Finalmente, los resultados se comparan con el español costarricense central, con otras variantes americanas, y con el español peninsular estándar.

La transcripción fonética está hecha en AFI, en nivel semiestrecho, y se utilizó para ella lo que parece ser una impresora matricial de no mucha resolución, como las que eran habituales en aquellos años.

Para esta muestra se ha escogido un pasaje del informante 2, hombre de entre 50 y 60 años, con educación primaria completa, de quien se nos dice que vivió fuera [de Puntarenas] dos años, probablemente en San José [de Costa Rica].

\subsection{Facsímil (Rodríguez Rojas 1992: 82)}

'ar'ummon'onde'kosaha'kike...

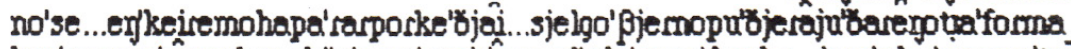

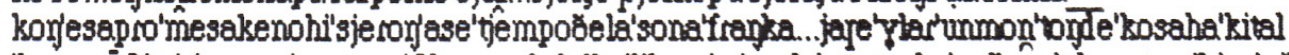

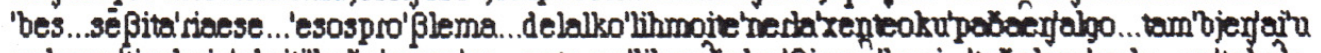
nakwes'tjonkejo'ekriti'kado'muco'es...patema'lihmobeho' jjemo'keai... 'boboloje'yaka ..en'bhela

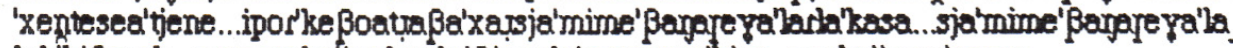
bh'bißereh...entonseha'jesdonde' $\beta$ jenela'suntotam'bjeg...porke'jome'pomo al'berdekeh'tanje ya'landolah'ksosahen'tonse...'Jome'sjentoeg. . ko je'dordemi'kasaime'pongosle'es

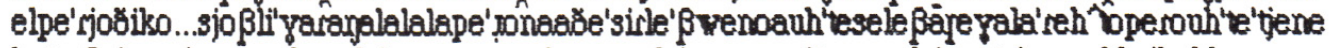

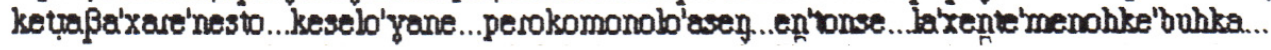

\subsection{Transcripción ortográfica}

Los problemas de Puntarenas

1 Hay un montón de cosas aquí que... no sé... en qué iremos a parar porque diay 2 si el gobierno pudiera ayudar en otra forma con esa promesa que nos hicieron 3 hace tiempo de la zona franca... y arreglar un montón de cosas aquí tal vez... 4 se evitaría ese... esos problemas... del alcoholismo y tener la gente ocupada 5 enalgo...tambiénhayunacuestiónqueyohecriticadomuchoes...paternalismo 6 del gobierno que hay... todo lo regala entonces la gente se atiene... y por qué 
7 voy a trabajar si a mí me van a regalar la casa... si a mí me van a regalar la... 8 los víveres... entonces ahí es donde viene el asunto también... porque yo me 9 pongo... al ver de que están regalando las cosas entonces... yo me siento en... 10 corredor de mi casa y me pongo a leer el periódico... si obligaran a la la la 11 persona a decirle bueno a usted se le va a regalar esto pero usted tiene que 12 trabajar en esto... que se lo gane... pero como no lo hacen... entonces... la gente 13 menos que busca... ${ }^{10}$

\subsection{Transcripción fonética adaptada}

1 [ 'ai 'un mon'ton de 'kosah a'ki ke | no 'se | en 'keiremoh a pa'rar porke 'ðjai | 2 sjel go'ßjerno pu'ðjeraju'ðar en ot.ıa 'forma $\mid$ kon 'esa pro'mesa ke noh i'sjeron 3 'ase 'tjempo ðe la 'sona 'franka | ja. ${ }^{\mathrm{z}} \mathrm{e}^{\prime}$ yla' run mon'ton de 'kosah a'ki tal 'bes | 4 se:ßita'ria ese | 'esos problema | del alko'lihmoi te'ner la 'xente oku'paða 5 en'algo|tam'bjen'ai 'unakwes'tjon kejo'ekriti'kaðo'mutso'es | paterna'lihmo 6 ðel go'ßjerno 'ke ai | 'toðo lo $\mathrm{I}^{\mathrm{z}} \mathrm{e}^{\prime}$ yala | en'tohe la 'xente se a'tjene | i por 'ke 7 ßo a traßa'xa. sja 'mi me 'ßay a $\mathrm{I}^{\mathrm{z}}$ eya'lar la 'kasa $\mid$ sja 'mi me ' $\beta a y$ a $\mathrm{s}^{\mathrm{z}}$ eya'la 8 loh 'bißereh | entonseh a'jes donde 'ßjene la'sunto tam'bjen | porke 'jo me 9 'pongo | al 'ber de keh'tan $\mathrm{I}^{\mathrm{z}}$ eya'lando lah 'kosah en'tonse | 'fjo me 'sjento en | 10 ko. $^{\mathrm{z}} \mathrm{e}^{\prime}$ dor de mi 'kasai me pongo a le'er el pe'rjoðiko | sjoßli'yaray a lalala 11 pe'.tona a ðe'sirle ' $\beta$ weno a uh'te se le $\beta a: \mathrm{x}^{\mathrm{z}}$ eyala'rehto pero uh'te 'tjene ke 12 tuaßa'xar e'nesto | ke se lo 'yane | pero komo no lo 'asen | en'tonse | la 'xente 13 'menoh ke 'buhka | ]

\section{PińERos 2006}

El lingüista colombiano Carlos Eduardo Piñeros ha creado uno de los más importantes recursos disponibles hoy para describir la variación de español en el mundo. Se trata de su página web Dialectoteca del español, construida durante sus años como profesor en la Universidad de Iowa. En ella se presentan grabaciones en audio y vídeo de 30 informantes distintos; de ellos, 8 son españoles y los 22 restantes proceden de 18 ciudades repartidas entre 11 países de América. Todos ellos son estudiantes universitarios o profesores jóvenes, tanto hombres como mujeres ${ }^{11}$.

\footnotetext{
${ }^{10}$ Transcripción basada en la original de la autora, aunque ajustada en algunos segmentos para restablecer la plena correspondencia con la versión fonética.

${ }^{11}$ Durante al año 2021 esta plataforma permanecerá inaccesible debido a la cancelación del software que se usó inicialmente para crearla. No obstante, tras contactar con su autor, y con las autoridades de la Universidad de Iowa, estas han informado que su actualización está en marcha desde el 1 de julio de 2021, y que se espera tenerla disponible de nuevo en la primavera de 2022.
} 
Cada módulo tiene cuatro elementos, de los que tres son habla espontánea y duran en torno a un minuto: Región, donde el informante describe su ciudad de origen; Anécdota, en el que narra algún suceso especial o divertido; y Opinión, donde explica su preferencia por algún escritor u obra literaria. Estas muestras aparecen en transcripción ortográfica. Pero hay una sección más, Cuento, en la que todos leen el mismo texto, el relato de 119 palabras "El viento del norte y el sol", bien conocido por los fonetistas de todo el mundo. Y de esta grabación se nos ofrecen dos transcripciones: la ortográfica -idéntica en todos- y la fonética, para la cual se utiliza el AFI en un nivel estrecho.

El valor de estas muestras para el estudio de la variación fonética es muy alto, porque con ellas se logra uno de los requisitos fundamentales del método experimental: que solo cambie lo que nos interesa que cambie, nada más. En efecto, todas las variables sociolingüísticas están aquí controladas, al pertenecer los informantes a un mismo segmento sociocultural y de edad. Y al leer todos ellos igual texto, desaparece también la variación gramatical o léxica (bien representada en los otros fragmentos), y solo queda la que afecta diatópicamente a la pronunciación. Se trata además de grabaciones de buena calidad de audio, y relativamente recientes (la compilación está fechada en 2006).

De la región que ahora nos ocupa, Piñeros contó con dos informantes: una hablante de San José de Costa Rica, y otro de David (Panamá).

\subsection{Facsimil (Piñeros 2006)}

Informante de San José (Costa Rica)

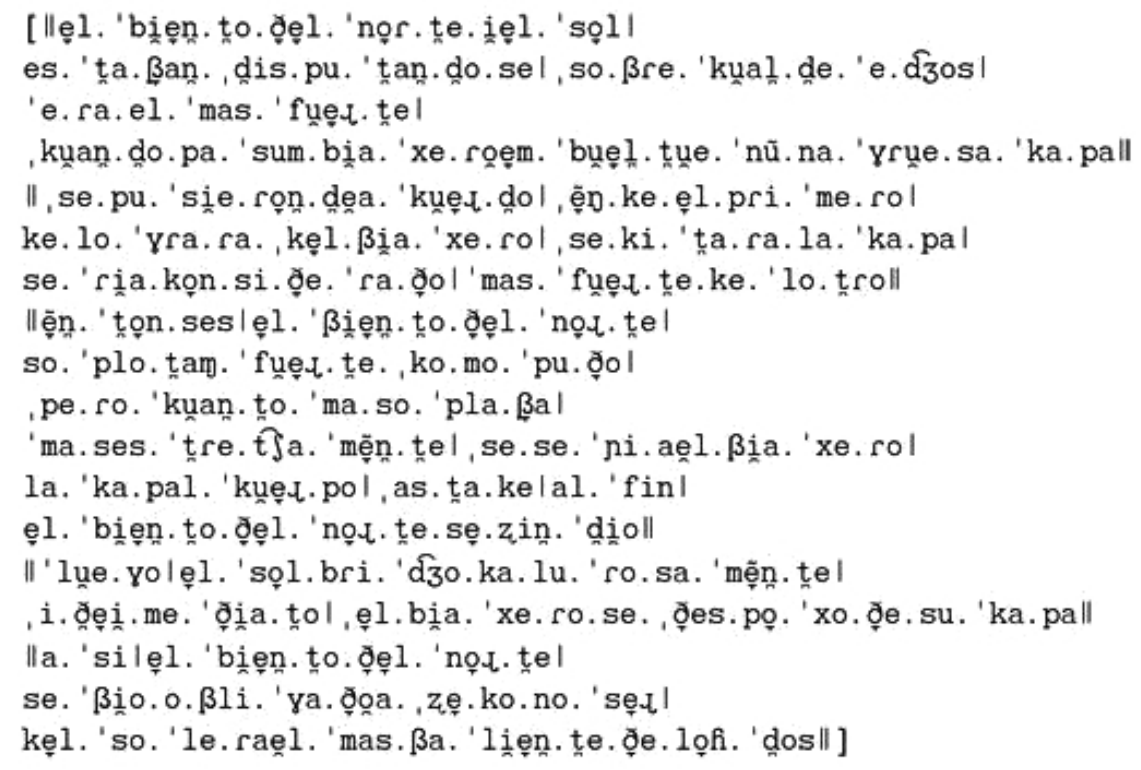


Informante de David (Panamá)

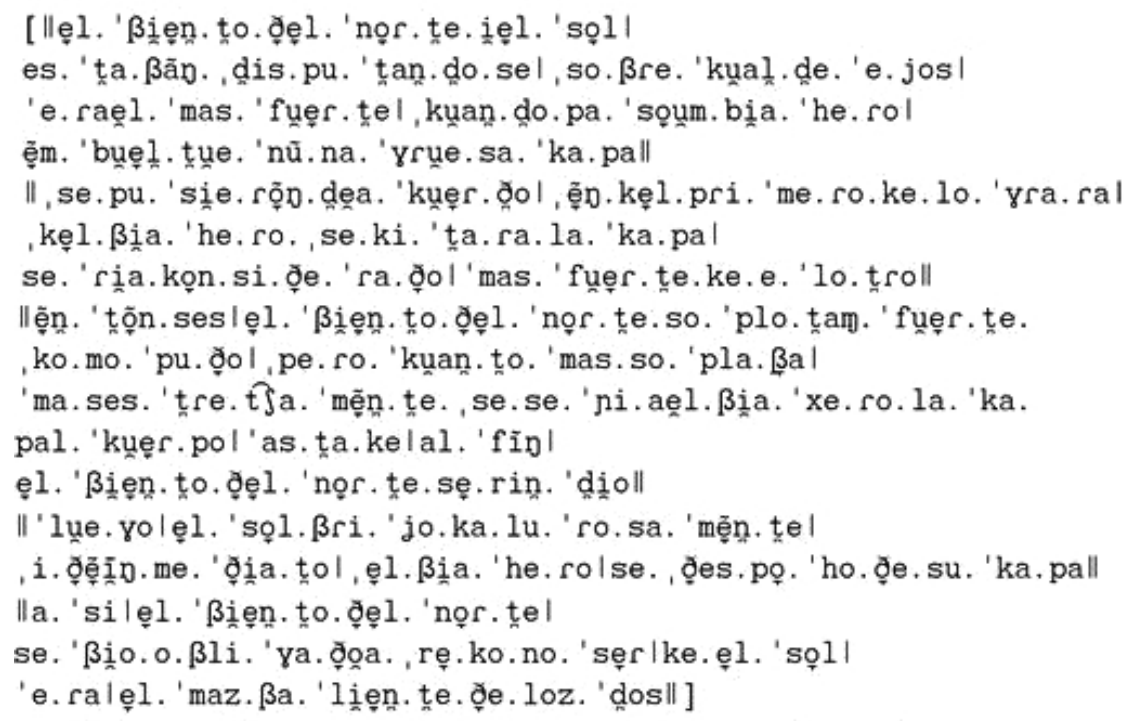

\subsection{Transcripción ortográfica}

El viento del norte y el sol

1 El viento del norte y el sol estaban disputándose sobre cuál de ellos

2 era el más fuerte, cuando pasó un viajero envuelto en una gruesa

3 capa. Se pusieron de acuerdo en que el primero que lograra que el viajero

4 se quitara la capa sería considerado más fuerte que el otro.

5 Entonces, el viento del norte sopló tan fuerte como pudo, pero cuanto

6 más soplaba más estrechamente se ceñía el viajero la capa al cuerpo

7 hasta que, al fin, el viento del norte se rindió. Luego, el sol brilló

8 calurosamente y de inmediato el viajero se despojó de su capa.

9 Así, el viento del norte se vio obligado a reconocer que el sol era

10 el más valiente de los dos.

\subsection{Transcripción fonética adaptada}

El viento del norte y el sol (informante de San José, Costa Rica)

1 [ el 'bjento ðel 'norte jel 'sol | es'taßan dispu'tandose | soßre 'kwal de 'ęjos |

2 'erael 'mas 'fwelte | kwando pa'sum bja'xero em'bweltwe'nuna 'yrwesa

3 'kapa || se pu'sjeron dea'kweldo | en ke el pri'mero | ke lo'yrara kel ßja'xero | 
4 se ki'tara la 'kapa | se'rja konsiðe'raðo | 'mas 'fwelte ke 'lotro || 5 en'tonses | el' $\beta$ jento ðel 'nolte | so'plo tam 'fwelte komo 'puðo | pero 'kwanto

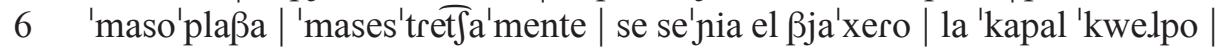
7 asta ke | al 'fin | el 'bjento đel 'nolte se rzin'djo || 'lweyo | el 'sol bri' jjo 8 kalu'cosa'mente | i ðeime'ðjato | el bja'xero se ðespo'xo ðe su 'kapa || 9 a'si | el 'bjento ðel 'nolte | se 'ßjo oßli' yaðoa rekono'sel | kel 'so'lera 10 el 'mas ßa'ljente ðe loh 'ðos ]

El viento del norte y el sol (informante de David, Panamá)

1 [ el 'ßjejto ðel 'norte jel 'sol | es'taßay dispu'tandose | soßre 'kwal de 'ejos | 2 'erael 'mas 'fwerte | kwando pa'soun bja'hero | em'bweltwe'nuna 'yrwesa 3 'kapa || se pu'sjeroy dea'kwerðo | en kel pri'mero ke lo'yrara | kel ßja'hero 4 se ki'tara la 'kapa | se'rja konsiðe'raðo | 'mas 'fwerte ke e'lotro || 5 en'tonses | el 'Bjento ðel 'norte so'plo tam 'fwerte komo 'puðo | pero 'kwanto

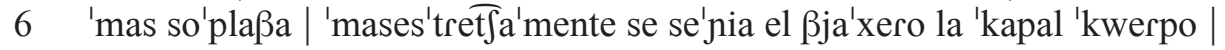
7 'asta ke | al 'fin | el 'Bjento ðel 'norte se rin'djo || 'lweyo | el 'sol ßri'jo 8 kalu'rosa'mente | i ðeinme'ðjato | el bja'hero | se ðespo'ho ðe su 'kapa || 9 a'si | el 'ßjento ðel 'norte | se 'ßjo oßli'yaðoa rekono'ser | ke el 'sol | 'era | 10 el 'maz ßa'ljente ðe loz 'dos ]

\section{ROBE 1960}

Entre 1943 y 1946, el hispanista norteamericano Stanley L. Robe pasó varias temporadas en diversas localidades rurales de las cuatro "provincias centrales" (Veraguas, Coclé, Herrera y Los Santos) de Panamá. Durante ese tiempo entrevistó a un total de 28 informantes, casi todos varones y escasa o nulamente letrados. Fruto de ese trabajo de campo fueron la monografía que ahora nos ocupa, centrada en fonética y morfología, y otra posterior (Robe 1963) dedicada a una recopilación ordenada de adivinanzas y pequeños poemas populares. El autor explica que su intención primordial originaria no era realizar un estudio lingüístico, razón por la cual no había previsto llevar ningún aparato de grabación, y, al coincidir su estancia con la guerra, tampoco lo pudo conseguir una vez allí. Sin embargo, confía en suplir la falta de registros de este tipo con las transcripciones que ilustran su capítulo sobre fonética, de las que aquí nos ocuparemos.

Para su recogida de materiales Robe manejó el Cuestionario lingüístico de Navarro, con una perspectiva dialectológica, pero en los quince años transcurridos hasta la elaboración de su estudio final lo enriqueció con un enfoque más estructuralista (al menos en la parte de fonética, pues la de morfología es más descriptiva). Como él explica, su objetivo, en consonancia con las corrientes lingüísticas europeas del momento, acabó siendo "...to determine broadly the phonemes of the dialect and identify with each its respective allophones" (Robe 1960: x). 
El autor utilizó para sus transcripciones un alfabeto fonético mixto, en el que predomina el ARFE junto a algunos símbolos y diacríticos del AFI (Robe 1960: xvii). Aquí se muestran tres textos: unas décimas transcritas a Remigio Graell, granjero retirado de Pocrí (Coclé), analfabeto, de 59 años; una adivinanza, obtenida de José María González, granjero de El Sesteadero (Los Santos), con estudios elementales, de 52 ańos; y unos versos tomados de José Natividad Quintero, granjero retirado de El Carate (Los Santos), analfabeto, de 102 años.

\subsection{Facsimil (Robe 1960: 157-158)}

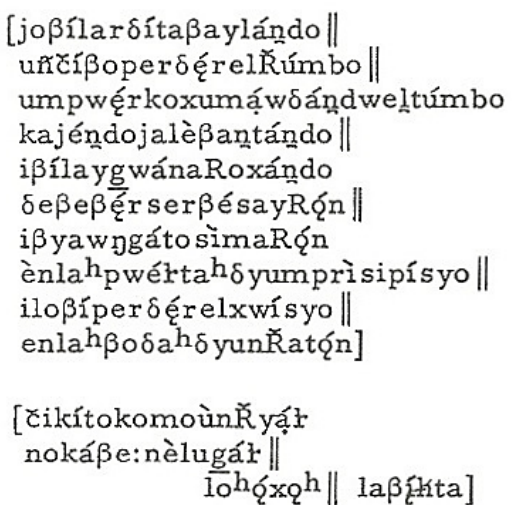

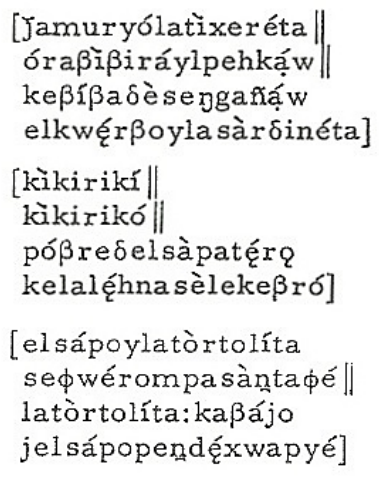

\subsection{Transcripción ortográfica}

\begin{tabular}{|ll|ll|}
\hline \multicolumn{3}{|c|}{ Décima (Coclé) } & \multicolumn{2}{c|}{ Versos (Los Santos) } \\
\hline 1 & Yo vi la ardita bailando, & 14 & Ya murió la tijereta, \\
\hline 2 & Un chivo perder el rumbo, & 15 & Ahora vivirá el pescado. \\
\hline 3 & Un puerco jumado dando el tumbo & 16 & Que viva desengañado \\
\hline 5 & Cayendo y alevantando & 17 & El cuervo y la sardineta. \\
\hline 6 & Y vi la iguana arrojando & & \\
\hline 7 & De beber cerveza y ron, & 18 & Quiquiriquí, \\
\hline 8 & En las puertas de un precipicio. & 20 & Pobre del zapatero \\
\hline 9 & Y lo vi perder el juicio & 21 & Que la lezna se le quebró. \\
\hline 10 & En las bodas de un ratón. & & \\
\hline & Adivinanza (Los Santos) & 22 & El sapo y la tortolita \\
\hline 11 & Chiquito como un real, & 23 & Se fueron para Santa Fe \\
\hline 12 & No cabe en el lugar. & 24 & La tortolita a caballo \\
\hline 13 & (Los ojos, la vista.) & 25 & Y el sapo pendejo a pie. \\
\hline
\end{tabular}




\subsection{Transcripción fonética adaptada}

\begin{tabular}{|c|c|c|c|}
\hline \multicolumn{2}{|r|}{ Décima (Coclé) } & \multicolumn{2}{|r|}{ Versos (Los Santos) } \\
\hline 1 & [ jo 'ßi lar'ðita ßaíl'lando | & 14 & fja mu'rjo la tixe'reta | \\
\hline 2 & un 'tfißo per'ðer el 'rzumbo | & 15 & 'ora $\beta i \beta i i^{\prime}$ rail peh'kau | \\
\hline 3 & um 'pwerko xu'mau 'ðandwel 'tumbo & 16 & ke ' $\beta i ß a$ ðesenga'nau \\
\hline 4 & ka'jendojaleßan'tando | & 17 & el 'kwerßoi la sarði'neta || \\
\hline 5 & i 'ßi la i' $\gamma w a n a r o ' x a n d o$ & & \\
\hline 6 & 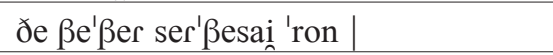 & 18 & kikiri'ki | \\
\hline 7 & i ßjaung 'gato sima'ron & 19 & kikiri'ko \| \\
\hline 8 & en la ${ }^{\mathrm{h}}$ 'pwelta ${ }^{\mathrm{h}}$ ðjum prisi'pisjo $\|$ & 20 & 'poßre ðel sapa'tero \\
\hline 9 & i lo 'ßi per'ðer el 'xwisjo | & 21 & ke la 'lehna se le ke' $\beta r o \|$ \\
\hline 10 & en $1 a^{\mathrm{h}} \beta$ oða ${ }^{\mathrm{h}}$ ðjun $\mathrm{r}^{\mathrm{z}} \mathrm{a}^{\prime}$ ton $\|$ & & \\
\hline & Adivinanza (Los Santos) & 22 & el 'sapoi la torto'lita \\
\hline 11 & t fji'kito komo un ' $r^{7}$ jal & 23 & se 'фwerom pa santa ' $\phi \mathrm{e} \mid$ \\
\hline 12 & no 'kaße:nelu'yal || & 24 & la torto'lita: ka'ßajo \\
\hline 13 & 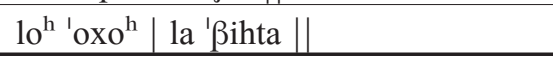 & 25 & ¿jel 'sapo pen'dexwa 'pje $\mid$ ] \\
\hline
\end{tabular}

\section{ConClusión}

El español hablado en América Central ha sido analizado en las últimas décadas con una atención excepcional, tanto en calidad como en cantidad, y de forma especialmente coordinada. No obstante, en lo que se refiere a la disponibilidad de textos fonéticos, si bien todos los aquí reunidos tienen un alto valor científico, siguen siendo ciertos al menos dos de los diagnósticos que hacía Quesada en 2006: por un lado, el aislamiento y falta de coordinación entre estas investigaciones; y por otro, el desequilibrio entre las zonas estudiadas, que en nuestro caso afecta especialmente a Guatemala y Honduras, de los que no hemos podido hallar hasta ahora ni un texto.

Sin embargo, tengo la esperanza de que la difusión de este trabajo pueda servir para conocer otros estudios aún pendientes de encontrar, y hacer más denso el mapa de estas muestras. Además, hoy existen vías al alcance de todos para sacar a la luz pública documentos que permanecen inéditos, y que de seguro también podrán enriquecer este y otros proyectos. Me refiero a los repositorios gratuitos para investigadores disponibles en Internet, como Researchgate y otros similares. Yo me permitiría animar a los directores y autores de esas tesis de licenciatura, de maestría o doctorales, valiosísimas pero tan a menudo sin publicar, para que simplemente las escaneasen y las colocaran en alguno de estos depósitos virtuales, con su debida y reconocida autoría, pero accesibles para cualquier interesado. 
De esa forma, todos los estudios que ahora se citan como "inéditos", y que muchas veces solo la buena suerte nos permite consultar, podrían en adelante ser citados con un enlace para su descarga, y alcanzarían igual vitalidad y relevancia que aquellos ya merecedores del "arduo honor de la tipografía", como diría Borges. Estoy seguro de que en esas páginas durmientes encontraríamos todos mucho que aprovechar, y desde luego algún que otro texto fonético se asomaría también de vez en cuando.

Por último, además de esta petición "retrospectiva”, querría formular otra más orientada al futuro, y es rogar a esos colegas que siguen promoviendo la investigación para que incluyan en el programa de los trabajos que tutelen un hueco para los textos fonéticos, esa herramienta descriptiva que, pese a haber sido orillada por algunas corrientes lingüísticas modernas, sigue siendo un elemento que todos necesitamos para completar nuestra visión de la variación del lenguaje.

\section{Obras citadas}

Componentes del Atlas Lingüístico de América Central (por paises)

\section{Belice}

Cardona Ramírez, Mauricio A. 2012. "Atlas lingüístico pluridimensional del español en Belice (ALEB). Nivel fonético". Bergen Language and Linguistics Studies 2.

Rivera Orellana, Erick. 2013. Atlas lingüistico-etnográfico pluridimensional de Belice (ALEB). Morfosintaxis. Léxico. San Salvador: UCA.

\section{Costa Rica}

Quesada Pacheco, Miguel Ángel. 2010a. Atlas lingüistico-etnográfico de Costa Rica (ALECORI). San José: UCR.

$$
\text { El Salvador }
$$

Azcúnaga López, Raúl E. 2012. "Atlas lingüístico-etnográfico pluridimensional de El Salvador (ALPES). Nivel fonético". Bergen Language and Linguistics Studies 2.

Rivera Orellana, Erick. 2011. Atlas lingüistico-etnográfico pluridimensional de El Salvador (ALPES): morfosintaxis, léxico. El Salvador: UCA.

\section{Guatemala}

Utgård, Katrine. 2006. Fonética del español de Guatemala. Análisis geolingüistico pluridimensional. Universidad de Bergen, Tesis de Maestría.

Chavarría Úbeda, Carmen \& Miguel Ángel Quesada Pacheco (s. p.). Atlas lingüistico-etnográfico de Guatemala (ALEG). Niveles morfosintáctico y léxico. In fieri.

$$
\text { Honduras }
$$

Hernández Torres, Ramón A. 2013a. Atlas lingüistico pluridimensional de Honduras (ALPH). Nivel fonético. Tegucigalpa: UNAH, Editorial Universitaria. . 2013b. Atlas lingüistico pluridimensional de Honduras (ALPH). Nivel morfosintáctico. Tegucigalpa: UNAH, Editorial Universitaria.

Ventura, Julio. 2013. Atlas lingüistico pluridimensional de Honduras (ALPH). Nivel léxico. Tegucigalpa: UNAH, Editorial Universitaria. 


\section{Nicaragua}

Chavarría Úbeda, Carmen \& María Auxiliadora Rosales. 2010. Atlas lingüístico etnográfico de Nicaragua (ALEN). Bergen: Universidad de Bergen.

$$
\text { Panamá }
$$

Cardona Ramírez, Mauricio A. 2012. "Atlas lingüístico pluridimensional del español de Panamá (ALEP). Nivel fonético". Bergen Language and Linguistics Studies 2.

Tinoco Rodríguez, Tarahy S. 2010. Atlas lingüistico-etnográfico de Panamá (ALEP): nivel morfosintáctico, nivel léxico. Bergen, Universidad de Bergen.

\section{Monografias, misceláneas y artículos}

Agüero Chaves, Arturo. 1962. El español de América y Costa Rica. San José: Lehmann.

Alarcos Llorach, Emilio. 1965. Fonología española. Madrid: Gredos.

Beardsley, Theodore S. 1993. "Necrology. Delos Lincoln Canfield (1903-1991)". Hispanic Review 61: 135-137.

Canfield, Delos Lincoln. 1960. "Observaciones sobre el español salvadoreño". Filología 6: 29-76.

. 1962. La pronunciación del español de América. Ensayo histórico-descriptivo. Bogotá: Instituto Caro y Cuervo.

1981. Spanish pronunciation in the Americas. Chicago: University of Chicago Press. 1988. El español de América: fonética. Barcelona: Crítica.

Caravedo, Rocío. 1989. El español de Lima: materiales para el estudio del habla culta. Lima: Pontificia Universidad Católica.

Fernández de Castro, Félix. 2007. "Estado actual y perspectivas del proyecto ATeFonHA (Antología de Textos Fonéticos Hispano-Americanos)”. En César Hernández y Leticia Castañeda, Eds. El español de América. Actas del VI Congreso Internacional (Tordesillas 2005). Valladolid: Diputación. 291-304. . 2010. "Capítulo 10. Fonética (antología y comentarios fonéticos)”. En Milagros Aleza y José María Enguita, Coords. La lengua española en América: normas y usos actuales. Libro electrónico, Valencia: Universidad de Valencia. 431-483.

. 2011. "Textos fonéticos de Puerto Rico y República Dominicana (1898-1983)". Moenia 17: 435-473.

. 2022. "Textos fonéticos de Cuba (1965-2006)". Nueva Revista de Filología Hispánica 70/1: 273-300.

García Mouton, Pilar, Coord. 2015. ALPI-CSIC [en línea], edición digital de Tomás Navarro Tomás, Dir. 1930-1954. Atlas Lingüistico de la Península Ibérica. Madrid: CSIC. Sitio Web: < www.alpi.csic.es > [18-dic-2018]

\& Isabel Molina Martos. 2017. Las hablas rurales de Madrid. Etnotextos. Bern: Peter Lang.

Navarro Tomás, Tomás. 1932. Manual de pronunciación española. Madrid: Junta de Ampliación de Estudios. 
. 1945. Cuestionario lingüistico hispanoamericano. Buenos Aires: Universidad de Buenos Aires.

Piñeros, Carlos Eduardo. 2006. Dialectoteca del español. The University of Iowa. Web: $<$ dialects.its.uiowa.edu > [19-oct-2018]

Quesada Pacheco, Miguel Ángel. 2008. "El español de América Central ayer, hoy y mañana”. Boletín de Filología 43/1: 145-74.

,Ed. 2010b. El español hablado en América Central: nivel fonético. Madrid / Frankfurt: Iberoamericana / Vervuert.

, Ed. 2013. El español hablado en América Central: nivel morfosintáctico. Madrid / Frankfurt: Iberoamericana / Vervuert.

Quilis, Antonio \& María José Quilis Sanz. 2002. El español en América, Madrid: Universidad Nacional de Educación a Distancia.

Robe, Stanley L. 1960. The Spanish of Rural Panama. Major Dialectal Features. Berkeley: University of California Press. . 1963. Hispanic riddles from Panama: collected from oral tradition. Berkeley: University of California Press.

Rodríguez Rojas, Alejandra. 1992. Análisis fonético y fonológico, nivel segmental, del español de la ciudad de Puntarenas. Universidad de Costa Rica, Tesis de Maestría inédita, dirigida por D. Víctor M. Sánchez Corrales.

Ruiz Hernández, Julio Vitelio. 1977. Estudio sincrónico del habla de Santiago de Cuba. Aspectos fonético y fonológico. Santiago de Cuba: Editorial Oriente.

Solano Rojas, Yamileth. 1986. "Una variación lingüística en el habla costarricense". Revista de Filología y Lingüistica 12.1: 133-143. 